\title{
Pilot Implementation of Health Information Systems: Issues and challenges
}

\author{
Bansler, Jørgen P.; Havn, Erling C.
}

Published in:

Proceedings of the 15th Americas Conference on Information Systems

Publication date:

2009

Document Version

Publisher's PDF, also known as Version of record

Link back to DTU Orbit

Citation (APA):

Bansler, J. P., \& Havn, E. C. (2009). Pilot Implementation of Health Information Systems: Issues and challenges. In Proceedings of the 15th Americas Conference on Information Systems (pp. Paper 510) $\mathrm{http}: / /$ aisel.aisnet.org/amcis2009/510/

\section{General rights}

Copyright and moral rights for the publications made accessible in the public portal are retained by the authors and/or other copyright owners and it is a condition of accessing publications that users recognise and abide by the legal requirements associated with these rights.

- Users may download and print one copy of any publication from the public portal for the purpose of private study or research.

- You may not further distribute the material or use it for any profit-making activity or commercial gain

- You may freely distribute the URL identifying the publication in the public portal

If you believe that this document breaches copyright please contact us providing details, and we will remove access to the work immediately and investigate your claim. 


\title{
Pilot Implementation of Health Information Systems: Issues and challenges
}

\author{
Jørgen P. Bansler \\ Technical University of Denmark \\ bansler@man.dtu.dk
}

\author{
Erling C. Havn \\ Technical University of Denmark \\ havn@man.dtu.dk
}

\begin{abstract}
Pilot implementation is a powerful and widely used approach in identifying design flaws and implementation issues before the full-scale deployment of new health information systems. However, pilot implementations often fail in the sense that they say little about the usability and usefulness of the proposed system designs. This calls for studies that seek to uncover and analyze the reasons for failure, so that guidelines for conducting such pilots can be developed. In this paper, we present a qualitative field study of an ambitious, but unsuccessful pilot implementation of a Danish healthcare information system. Based on the findings from this study, we identify three main challenges: (1) defining an appropriate scope for pilot implementation, (2) managing the implementation process, and (3) ensuring commitment to the pilot. Finally, recommendations for future research and implications for practice are provided.
\end{abstract}

\section{Keywords}

Healthcare information systems, pilot implementation, information systems development

\section{INTRODUCTION}

Design and implementation of new health information systems (HIS) is an extraordinarily complex and dynamic sociotechnical process in which difficult technical and organizational problems are confronted within a conflict-laden arena (Berg et al. 2003). Ambiguity and uncertainty are salient aspects of this process and it is, therefore, "fundamentally unfit for a strict planning and controlling approach" (Berg 2004, p. 183). Instead, HIS requires a flexible, iterative and incremental approach that emphasizes the need for continuous learning, and embraces improvisation and experimentation (Berg et al. 2003, Berg 2004, Heeks 2006). HIS development projects, therefore, often involve a pilot implementation of the system under development to evaluate its usability and usefulness and to gauge potential organizational issues that might arise from its implementation (See e.g. Berg 2004). However, there are surprisingly few studies of how such pilot implementations are conducted and of the difficulties they may incur.

This paper presents findings from a longitudinal, qualitative field study on the conduct of an ambitious, but unsuccessful, pilot implementation of a boundary spanning IS system within the Danish healthcare sector. Based on these findings, we identify the following main challenges that systems developers face when undertaking a pilot implementation of a new information system: (1) defining the scope of the pilot implementation, (2) managing the technology and its use within one or more work settings, and (3) ensuring commitment from pilot users and their managers.

\section{BACKGROUND}

Within the fields of information systems development and software engineering it has long been acknowledged that systems development is a learning process that requires an iterative approach, so that feedback loops between successive stages of development (e.g. requirements definition, systems design, implementation, and operation) are facilitated (Boehm 1988, Sommerville 2004). There are several ways in which one can facilitate an iterative design process and at the same time actively involve users. Two of the most effective and widespread techniques are prototyping and pilot implementation.

A prototype is a model or an initial version of an information system that is used to elicit requirements, demonstrate design concepts, explore particular solutions, and generally, "to find out more about the problem and its possible solutions" (Sommerville 2004, p. 409). By working with the prototype hands-on, users can assess the system's strengths and weaknesses and explore how well it supports their work. However, as Sommerville (2004) also points out, a general limitation on prototyping is that "the mode of use of the prototype may not correspond with how the final delivered system is used" (p. 412). Prototypes are usually tested within a laboratory setup, and there may be significant differences between the artificial lab setting and the real-life work setting where the final system is going to be used. This weakness has led to the growing use of pilot implementation as a complement to prototyping. 
In a pilot implementation or field trial, a group of test users agree to use the system on a trial basis, but in their actual work environment and with real data. Ideally, they simultaneously experiment with adjusting their work processes to capitalize on the affordances offered by the technology. The job of the system developers is to observe the results, collect feedback from users, and identify opportunities for improving the system design and the implementation process (Lauesen 2004).

Normally, the purpose of a pilot implementation is threefold: (1) it can contribute to identifying and prioritizing the final system requirements, based on practical experience with its use in a real-life organizational setting; (2) it can help identify necessary or desirable changes in the work organization and processes affected by the system; and (3) it can produce valuable insights for implementing the system, if it comes to full-scale deployment.

The principle of pilot implementations is that the new system is tested within part of an organization, with operational data, for a certain period of time. This is often difficult and costly, because it requires, first, that the system under development is to a large extent functional and, second, that there is a concerted effort to integrate it into the users' work practices so that it becomes part of their normal work routines. This is necessary, because it is only when the pilot users have become familiar with the system and use it daily as part of their everyday work routines that it will be possible to say anything substantial about its strengths and weaknesses (Leonard-Barton 1988; Orlikowski 1995).

It is particularly challenging to carry out pilot implementations of HIS, because they usually span several organizational units and are intended to connect users across organizational and professional boundaries. The users are therefore typically a very heterogeneous group of people who need to share information, but who otherwise work in different contexts, often without knowing each other. The organizational complexity of this not only complicates the system design, it also makes it more difficult to succesfully implement the pilot, because the system must simultaneously be integrated in a controlled and coordinated way into a number of different organizational units and work practices. Thus, it is essential that system developers and the various groups of pilot users work systematically on the mutual adaptation of the system and organization during the period of pilot implementation.

\section{CASE STUDY}

We studied the pilot implementation of a national electronic Pregnancy Record (ePR) in a county in Denmark. We used a qualitative case study approach in an in depth exploration of the issues and difficulties involved in its implementation (Walsham 1995, 2006). We followed the development of the ePR for over more than two years, i.e. from early in the design phase until the end of the pilot implementation. Our primary data collection methods were observations and semi-structured interviews. We conducted a total of 38 interviews, and detailed notes were taken at all meetings and observations. All interviews were recorded and transcribed. In addition to the observations and interviews, we reviewed relevant documents from the project (e.g. requirements specifications, system descriptions and implementation plans). To corroborate our findings we shared our preliminary findings with key persons (pilot users and project group members), and they provided helpful comments that confirmed and helped us elaborate our conclusions.

In the following we will briefly describe the design of the ePR and the course of events following its introduction into the healthcare system.

\section{The electronic Pregnancy Record (ePR)}

In Denmark, care during pregnancy and in childbirth is usually organized as a shared care arrangement involving the woman's general practitioner (GP), a midwives' clinic and a public hospital. Shared care implies that the responsibility for the health of the woman and her baby is shared, and it requires good communication between all parties involved. To this end, the National Board of Health has decreed that a common paper form - the Pregnancy Record (PR) - must be used for all women enrolled in the shared care program. The woman's GP commences the PR, and the record is given to the woman so that she can bring it with her to all subsequent appointments during her pregnancy, including those with the midwife and at the hospital. At each visit, the care provider must record in the PR pertinent information concerning diagnostic and treatment decisions.

As part of the Danish government's general drive to promote the use of electronic communication within the healthcare system, it was decided to develop a web-based electronic pregnancy record (ePR) as a substitute for the paper based record. The aim was to enhance communication and information sharing and thereby improve continuity of care as well as increase the pregnant woman's participation in her own care. Given that the system would be Internet-based, it was assumed that it would provide easy access to pregnancy records wherever and whenever needed.

The ePR application was developed by a private software firm in close cooperation with the Danish national ehealth portal, Sundhed.dk, and two counties, which had volunteered to be the first to pilot implement the application. The development of the ePR began in early 2004 and about a year later the first version of the software was delivered and ready for the pilot implementation. 


\section{The pilot implementation}

The pilot implementation was planned to last 12 months and involve 3 general practices (with a total of 10 GPs), one midwives' clinic, the department of obstetrics at a public teaching hospital and approximately 100 women. The purpose of the pilot was to examine the usability and usefulness of the application in actual clinical work as well as to solicit ideas for design improvements from healthcare providers and the women participating in the trial.

The pilot implementation was organized in the following way: The ePR software was running on Sundhed.dk's servers, and both the clinical users and the participating women would connect to it from their local PCs via the Internet. Sundhed.dk had the overall responsibility for the trial, whereas the county was responsible for training clinical users, setting up PCs, and all other local arrangements. The software provider was in charge of maintaining and upgrading the ePR software during the pilot period.

The local organization in the county consisted of a project group with participants from the obstetrics department, the midwives' clinic and each of the three general practices. An IT consultant acted as the project manager and took care of the day-to-day running of the pilot project. The participating GPs were responsible for recruiting the women and for creating an electronic pregnancy record during their first appointment. Simultaneously, the GPs would refer the selected women to the midwives' clinic and to the obstetrics department for subsequent appointments and tests.

The GPs enrolled the first women in the trial and created the first electronic pregnancy records in October 2005. During the first couple of months the healthcare providers often experienced technical breakdowns, delays and incomprehensible errors when using the application. Sundhed.dk and the software provider managed to solve the majority of these problems and stabilize the operation of the central servers by early 2006, but by that time many pilot users had become very frustrated and had decided, or at least threatened, to withdraw from the pilot implementation. By February 2006 three of the GPs had left the project, and the department of obstetrics was also considering to pull out. Two months later, in May, the county decided to stop the enrollment of new women in the pilot and to phase out the trial.

The many technical problems, the users' growing frustrations, their waning commitment, and the premature ending of the trial rendered the outcome of the pilot implementation indeterminate and inconclusive. None of the sites (the general practices, the midwives' clinic, and the obstetrics department at the hospital) managed to successfully appropriate the new technology and to fit it into their individual and collaborative working practices. The users never reached a point where they were able to use the application routinely, on a day-to-day basis, and they never gained enough experience to become proficient users. The bottom line was that not much could be said about the questions that the trial was supposed to address. Little could, in particular, be said about what it would take to integrate it into existing working practices or how it would affect the overall work organization. Moreover, little could be said as to whether it would have the potential to facilitate cross-border communication and improve the quality and continuity of care.

\section{ANALYSIS OF THE CASE STUDY FINDINGS}

Based on a detailed review of the implementation process, we have identified three major difficulties that complicated the pilot project and eventually led to its failure.

\section{Defining the scope}

The project had difficulties defining an appropriate scope for the pilot implementation. As a result many pilot users - in particular, the nurses and doctors at the hospital - had only sporadic contact with the ePR and never became proficient users. This happened in spite of the fact that the local project group (in the county) was very much aware of the problem with "sporadic" users and deliberately tried to design the field trial so that it involved as few pilot users as possible and so that each pilot user would use the system regularly, thus, helping to familiarize them with it.

The project group limited the number of GPs participating in the trial to 10, and furthermore made sure that the women participating in the trial would be referred to the same few selected midwives (a group of 5 persons) at the midwife's clinic. However, when it came to the hospital, it proved impossible to organize the pilot so that it would only include a few doctors and nurses. The organization of hospital work - with its demand for continuous round-the-clock operation, rotating shiftwork schedules, and many unanticipated events - simply made it impossible to limit participation to a small group of users.

The local project manager in the county knew this was a predicament, but she realized that the only way of solving the problem of "sporadic" users at the hospital would have been to increase the number of pregnant women enrolled in the pilot, thereby increasing the frequency of meeting a woman with an electronic pregnancy record. Given that the hospital manages more then 3.500 births per year, the number of women participating in the trial would have had to have been increased dramatically (maybe to 1.000 women or more) to really make a difference. This, in turn, would have required the involvement of far more midwives and general practitioners (at least 100 more), and it would have been exceedingly costly. Moreover, the project would have looked more like a full-scale implementation.

As expected, the predominance of "sporadic" users at the hospital created significant problems. Most doctors and nurses dealt very infrequently with women participating in the trial, and they never developed work routines with the ePR. Consequently, 
they found it difficult to use, and they tended to view it as an irritating nuisance imposed upon them by some outside authority. The nurses often "forgot" to read and write in the ePR, and in many cases the doctors outright refused to do so. Clearly, this extensive non-use had a detrimental effect on the pilot implementation. It made evaluating the ePR's usability and usefulness in the hospital setting impossible, and the resulting incompleteness of the electronic records also severely diminished the potential value of the ePR to the midwives and the general practitioners participating in the trial.

\section{Managing the implementation process}

The pilot project was plagued by technical problems from the beginning, and the project organization turned out to be unable to deal effectively with them. This inability to cope with unanticipated difficulties had a significant negative impact on the process and outcome of the pilot implementation. As it turned out, many pilot users, in particular some of the GPs, had trouble accessing the electronic pregnancy record via the Internet and they were often "thrown off" by the server in the middle of a session. The root causes of these technical problems turned out to be quite difficult to identify and some of them were not solved until the third software release in February 2006, after more than four months of operation.

Another important problem was the hospital's insufficient number of PCs, and the fact that they were often in rather impractical locations. For instance, a nurse performing an ultra-sound scanning would have to run back and forth between the scanning apparatus and the PC, because the machines were placed in two different rooms. Similarly, only a few of the doctors' consulting rooms were equipped with PCs.

Finally, the pilot introduced the use of digital signatures at the midwives' clinic and the hospital for the first time, and this proved to be much more problematical than originally expected. For security reasons, each pilot user was issued a memory card with their digital signature. To access the ePR, they had to insert this card into a USB reader and type their personal password. However, quite often pilot users (particularly the hospital clinicians) had either never obtained a valid digital signature, or they had lost their memory card somewhere, or forgotten it at home. In some instances, they then borrowed a card and a signature from one of their colleagues. In other cases, they simply gave up using the ePR. In both instances, the outcome was corrupted (i.e. incorrect or incomplete) electronic records

The trouble was not so much that the project encountered a number of technical and organizational problems (this is, after all, exactly what you would expect in a pilot implementation and, one might add, its raison d'être), but rather that the project organization was unable to react quickly and effectively to resolve them.

Firstly, the technical problems often took far too long to identify and correct. In most cases, solving the problem required the involvement of both Sundhed.dk and the software company, but communication between the local project group in the county, Sundhed.dk and the private software company did not function well. Furthermore, disagreements between Sundhed.dk and the software company as to who was responsible for solving a given problem lead to further delays. Not surprisingly, this slow and inept handling of the technicalities produced tremendous frustration and dissatisfaction among the test users

Secondly, the project group had neglected to appoint people responsible for organizing and facilitating the pilot implementation locally. In the midwives' clinic, the head midwife undertook this task on her own accord, and in two of the general practices, one of the GP's informally assumed responsibilities for implementing the system and helping the others. However, this kind of voluntary action did not happen at the hospital, and as a result, even small problems ended up having a large impact on the process and outcome of the pilot implementation.

\section{Ensuring commitment from users and their managers}

The pilot project had difficulty gaining and, in particular, maintaining commitment to the trial among users and their managers. Before the trial began, most pilot users expressed a positive interest in it, but as the process moved forward and the participants became aware of the amount of extra work required, their commitment, in practice, dwindled. The ePR project at the hospital was, for instance, given a low priority because it was just a pilot and not a "real" project. So, at times when they felt that their workload was too great, the clinicians frequently gave up struggling with the ePR.

The many technical problems in the beginning and the project's slow and inadequate response further eroded the pilot users' commitment. They could not understand why it took so long to solve the technical problems, and they felt that project management did not consider their complaints and suggestions important. One of the GPs described his experience in the following way:

And it's been frustrating for us, because if I'm going to test something, then I need some quick feedback. So that it can be fixed within a few weeks so that we can try it again, and see how it works. But then we could see right away that no matter what we came up with, it was... It wasn't relevant.

As this quote indicates what exaperated and demotivated the pilot users was first of all the feeling of not being taken seriously. This particular GP had often participated in the pilot testing of new IT systems, and he was prepared for difficulties, complications and extra work, and in a sense more likely to be responsive to the pilot than so many other of his colleagues. However, the feeling that he was not taken seriously and that his feedback did not really matter made him angry. 


\section{DISCUSSION}

We began this paper by noting that while pilot implementation is of great practical importance, it has sparked little interest amongst researchers, and - despite the fact that pilot implementations often fail - little is known about the reasons for failure and how to avoid it. Our position is that pilot implementation is a very useful and highly recommendable development technique, but also that it is very difficult to ensure success, particularly within complex organizational settings such as within healthcare. We think that there is a pressing need to critically assess current practices and to reflect upon the reasons why many pilot implementations are less than successful. Only in this way can we improve practice.

\section{Implications for practice}

Drawing from our analysis, we identify three important challenges that developers must cope with when engaging in pilot implementation of an information system:

The first, and most important, challenge is to define an appropriate scope for the pilot implementation. In other words attending to questions such as, how many organizational units and how many users from each unit should participate, and for how long should the implementation last?

For cost reasons, the scope of the pilot implementation should be as limited as possible, but at the same time one must ensure that the pilot fulfills its purpose and is somehow representative of the larger scale implementation. In particular, one should take care to define the scope of the pilot so that it is possible to achieve normal routine operation of the system for a sustained period of time, and so that the pilot users become familiar with the system and learn to integrate it in their daily clinical work. Otherwise, the pilot implementation will be virtually worthless. In some cases, identifying an appropriate scope may prove to be impossible, and in such cases it may be better to simply drop the pilot implementation and find alternative ways of proceeding (e.g. by relying more heavily on prototyping and incremental development).

The second major challenge is to manage the technology and facilitate its implementation in the each of the involved organizations. This is an ongoing process that involves coping with unanticipated technical problems as well as training users, integrating the technology in the various work practices, and reorganizing work to make the most of the new technology. Based upon our case analysis, we can make a number of recommendations:

- The system should be technically well tested and to a large extent operational prior to pilot implementation.

- The pilot project should be organized in such a way that it can attend to the unforeseen technical problems that inevitably will show up, especially in the beginning of the implementation process, despite best efforts to test the system in advance. Clear demarcations of responsibility and lines of communication must be established, and the necessary resources must be allocated to solve the problems quickly.

- Finally, after the initial implementation of the technology, a substantial and sustained commitment of organizational resources to assimilate the system into the pilot users' work practice is critical. The integration of the system into the day-to-day organizational routines requires an ongoing mutual adaptation of technology and organization. One way of achieving this could be by appointing a small group of organizational members - for instance users with an interest in new technology - as "mediators" (Orlikowski et al. 1995) or "gardeners" (Nardi \& O'Day 1999), responsible for customizing the system, assisting their co-workers in using it, and promoting changes in procedures and work organization.

The third challenge is to ensure commitment from managers, users, and other stakeholders involved in the pilot. There is always the risk that the pilot implementation will loose out to other priorities, because it is "just an experiment" and, thus, less important than the organization's core obligations. There are also a number of lessons that can be learned from this case:

- It must be recognized that participating in a pilot implementation requires extra work from the involved users, and that sufficient resources, e.g. in the form of extra people, have to be allocated to the participating organizational units during the pilot.

- Feedback from pilot users, both in form of complaints or suggestions for improvements, should be taken seriously and acted upon quickly. Otherwise, users may feel overlooked and loose motivation to participate in the project

- It is important to actively manage expectations about the intent, process and outcome of the pilot implementation. In other words, system developers should communicate openly and candidly with users and other stakeholders so that all parties understand what can and cannot be done with the time and resources available.

\section{Implications for future research}

Although these issues and recommendations are relatively general and likely to apply in many situations, much could probably be learned by examining the process and outcome of pilot implementations in other organizational contexts and with other technologies and applications. The challenges and issues related to pilot implementation probably vary with the type of IT systems and organizations involved. It is probably more difficult to carry out pilot implementations of IT systems that span several organizational and professional boundaries than systems, which are mainly intended for a single or a few 
organizational units or a single occupational group. Similarly, organizational aspects such as size, culture, and organizational slack are likely to influence the process and outcome of pilot implementations.

Further studies could explore the importance of these specific characteristics and identify under what circumstances pilot implementations are likely to be successful. We also need a more thorough understanding of how pilot implementation can be set up and managed so as to be useful and valuable. Further research is needed on how different ways of organizing and conducting pilot implementations effect their success, i.e. on unearthing how pilot implementation is dependent on the specific organizational and technological conditions.

\section{CONCLUSION}

Researchers and practitioners increasingly recognize that the design and implementation of HIS is a highly complex sociotechnical process, which requires an iterative approach, based on active user involvement and the use of experimental techniques such as prototyping and pilot implementation.

In this paper we have focused on the use of pilot implementations to identify and prioritize the final system requirements, based on practical user experience, and to identify necessary or desirable changes in the clinical work organization and processes around the system. We believe pilot implementations have much merit as an approach to systems design when system performance is highly contingent on context. However, while the systematic use of pilot implementations undoubtedly can lead to significant improvements in systems design, such pilots are also costly and difficult to perform, because they require a commitment to implement and use the system in clinical practice over a sustained period of time. Pilot implementations of HIS are particularly difficult to carry out because of the inherent organizational complexity of the healthcare system and because of the fact that most HIS involve several organizational units and are intended to be used by many different types of users with different professional backgrounds and for different work tasks and purposes.

\section{ACKNOWLEDGEMENTS}

The authors are grateful to the GPs, midwives, hospital clinicians and pregnant women who let us observe their work and interview them. The Danish Council for Strategic Research provided financial support for the study.

\section{REFERENCES}

1. Berg, M. (2004). Health Information Management. Integrating Information Technology in Health Care Work. Routledge, London, UK.

2. Boehm, B.W. (1988). A spiral model of software development and enhancement. IEEE Computer May 1988, pp. 61-72.

3. Bossen, C. (2007). Test the artefact—develop the organization: the implementation of an electronic medication plan. International Journal of Medical Informatics 76, pp. 13-21.

4. Gulliksen, J., B. Göransson, I. Boivie, S. Blomkvist, J. Persson \& Å. Cajander (2003). Key principles for user-centred systems design. Behaviour \& Information Technology 22 (6), pp. 397-409.

5. Heeks, R. (2006). Health information systems: failure, success and improvisation. International Journal of Medical Informatics 75, pp. 125-137.

6. Klein, H.K. and M.D. Myers (1999). A set of principles for conducting and evaluating interpretive field studies in information systems. MIS Quarterly 23, pp. 67-94.

7. Leonard-Barton, D. (1988). Implementation as Mutual Adaptation of Technology and Organization. Research Policy 17, pp. 251-267.

8. Lauesen, S. (2002). Software Requirements. Addison-Wesley, London, UK.

9. Nardi, B.A \& V.L. O’Day (1999). Information Ecologies. MIT Press, Cambridge, MA.

10. Orlikowski, W.J., J. Yates, K. Okamura \& M. Fujimoto (1995). Shaping electronic communication: the metastructuring of technology in the context of use. Organization Science 6 (4), pp. 423-444.

11. Pilemalm, S. \& T. Timpka (2008). Third generation participatory design in health informatics - making user participation applicable to large-scale information systems projects. Journal of Biomedical Informatics 41, pp. 327-339.

12. Sommerville, I. (2004). Software Engineering. Pearson, Essex, UK.

13. Walsham, G. (1995). Interpretive case studies in IS research: nature and method. European Journal of Information Systems 4:74-81.

14. Walsham, G. (2006). Doing interpretive research. European Journal of Information Systems 15, pp. 320-330. 